\title{
Elemental Compositions and Chemical Mass Closure of Fine Particulate in an Animal Feeding Operation Facility and Its Vicinity
}

\author{
Qian-Feng Li', Lingjuan Wang-Li2 ${ }^{*}$, R. K. M. Jayanty ${ }^{3}$, Sanjay Shah ${ }^{2}$ \\ ${ }^{1}$ Department of Animal Science, Michigan State University, East Lansing, USA \\ ${ }^{2}$ Department of Biological and Agricultural Engineering, North Carolina State University, Raleigh, USA \\ ${ }^{3}$ RTI International, Research Triangle Park, USA \\ Email: ${ }^{\text {Iwang5@ @ncsu.edu }}$
}

Received 27 February 2015; accepted 29 April 2015; published 4 May 2015

Copyright (C) 2015 by authors and Scientific Research Publishing Inc.

This work is licensed under the Creative Commons Attribution International License (CC BY). http://creativecommons.org/licenses/by/4.0/

\section{(c) (i) Open Access}

\begin{abstract}
Particulate matter (PM) emissions from animal feeding operations (AFOs) have been considered as an important contributor to ambient PM in rural areas. Investigation of the chemical compositions of $\mathrm{PM}_{2.5}$ inside and in the vicinity of AFOs can enhance our understanding of the AFO emissions impact on ambient PM characteristics. This year-long field study was conducted on a commercial egg production farm to investigate ambient PM chemical compositions as impacted by the air emissions from the production houses. The $\mathbf{P M}_{2.5}$ samples were collected from five sampling stations (one in-house station and four ambient locations in four wind directions). The trace elements, major ions, organic carbon (OC) and element carbon (EC) were analyzed by X-ray florescence (XRF), ion chromatography (IC), and thermo-optical analyzer, respectively. There were significant differences in elemental compositions between PM samples from in-house station (ST1) and ambient stations (ST2-ST5). The chemical mass balance analysis revealed that OC accounted for above $50 \%$ of $\mathrm{PM}_{2.5}$ mass at in-house and ambient stations; $\mathrm{NH}_{4}^{+}, \mathrm{SO}_{4}^{2-}$, and $\mathrm{NO}_{3}^{-}$accounted for about $40.0 \%$ of the total $\mathbf{P M}_{2.5}$ mass in ambient locations and for only $12 \%$ of the total $\mathbf{P M}_{2.5}$ mass in house. The measured $\mathbf{P M}_{2.5}$ masses agreed with the sums of the masses of chemical compositions at all stations except for the in-house station. Knowledge gained from this study, with additional consideration of $\mathrm{NH}_{3}$ concentrations and emissions, will lead to better understanding of $\mathbf{P M}_{2.5}$ source and formation, fate and transport, and their atmospheric dynamics.
\end{abstract}

\section{Keywords}

Animal Feeding Operations, $\mathbf{P M}_{2.5}$, Chemical Speciation, Elemental Composition, Mass Balance

\footnotetext{
"Corresponding author.
}

How to cite this paper: Li, Q.-F., Wang-Li, L.J., Jayanty, R.K.M. and Shah, S. (2015) Elemental Compositions and Chemical Mass Closure of Fine Particulate in an Animal Feeding Operation Facility and Its Vicinity. Journal of Environmental Protection, 6, 409-425. http://dx.doi.org/10.4236/jep.2015.65040 


\section{Introduction}

As a criteria pollutant, $\mathrm{PM}_{2.5}$ is of increasing concern. Studies have demonstrated evidence of its negative health effects on human and animals [1]-[4]. Korrick et al. [2] found that $\mathrm{PM}_{2.5}$ was associated with significant effects on pulmonary function in adults during prolonged outdoor exercise. $\mathrm{PM}_{2.5}$ can also adversely impact visibility, and contribute to acidification of lakes and streams, thereby degrading ecosystem health [5] [6].

Fine particulate matter (i.e., $\mathrm{PM}_{2.5}$ ) can originate from anthropogenic and natural sources or form through chemical reactions [7]. Different areas and regions have different major emission sources, climatology and geography, and therefore wide temporal and spatial variation in $\mathrm{PM}_{2.5}$ concentrations and characteristics are expected. Because of its relatively long residence time ( 3 - 5 days) in the atmosphere, $\mathrm{PM}_{2.5}$ may transport large distances from emission sources [7]. Geological materials, e.g. oxides of $\mathrm{Al}, \mathrm{Si}, \mathrm{Ca}, \mathrm{Ti}$ and $\mathrm{Fe}$, organic matter, elemental carbon, sulfate $\left(\mathrm{SO}_{4}^{2-}\right)$, nitrate $\left(\mathrm{NO}_{3}^{-}\right)$and ammonium $\left(\mathrm{NO}_{4}^{+}\right)$are six major components accounting for majority of $\mathrm{PM}_{2.5}$ mass [8]. The water-soluble ions $\left(\mathrm{SO}_{4}^{2-}, \mathrm{NO}_{3}^{-}, \mathrm{NO}_{4}^{+}\right.$, etc.) in many areas account for a major fraction of $\mathrm{PM}_{2.5}$ mass [9]. The ionic compositions of $\mathrm{PM}_{2.5}$ link to various chemical reactions in aerosols [10]. The chemical characteristics provide basic information for studies of $\mathrm{PM}_{2.5}$ source apportionment and assessment that may lead to development of cost effective mitigation strategies.

Particulate matter (PM) emissions from animal feeding operations (AFOs) have been considered as an important contributor to ambient $\mathrm{PM}$ in rural areas. Knowledge about the chemical compositions of $\mathrm{PM}_{2.5}$ inside and in the vicinity of AFOs can advance our understanding of the AFO air emission impacts on ambient PM characteristics, and the fate and transport of air pollutants associated with AFOs. While studies have been done to characterize elemental and/or ionic compositions of PM in animal production facilities [11]-[13], research gaps remain in developing AFO PM chemical composition profiles for source apportionment. Moreover, temporal and spatial variations of AFO PM chemical compositions are not well understood. In this study, a field investigation was conducted to characterize the trace elements and other chemical compositions of $\mathrm{PM}_{2.5}$ inside and in the vicinity of a commercial egg production farm. The objectives were to: 1) provide trace elemental composition dataset of $\mathrm{PM}_{2.5}$ in AFO areas; 2) assess contributions of different chemical compositions to the total mass of the $\mathrm{PM}_{2.5}$ through mass closure analysis. Information gained from this research may help to develop AFO $\mathrm{PM}_{2.5}$ source profile to assess the role of AFOs on ambient air quality in agriculture intensive rural areas, which is absence in US EPA's national ambient air quality monitoring networks.

\section{Methodology}

\section{1. $\mathbf{P M}_{2.5}$ Sampling Station}

The field sampling of $\mathrm{PM}_{2.5}$ was conducted on a commercial egg production farm in North Carolina, USA. The egg farm had nine production houses with capability of housing approximate one million laying hens. More information about the production farm may be found in Wang-Li et al. [14].

Samples of $\mathrm{PM}_{2.5}$ were simultaneously taken at five stations with one in a production house (ST1), and the other fours at ambient locations (ST2-5) surrounding the farm nearby the property lines of the egg production farm (Figure 1). Five Partisol (Model 2300, Thermo Scientific) $\mathrm{PM}_{2.5}$ speciation samplers were used to take the samples for 24-hr durations. The samplers were equipped with three cartridges, of which one was the Teflon filter cartridge for $\mathrm{PM}_{2.5}$ mass and element analysis, one Nylon filter cartridge for ion analysis, and one Quartz filter cartridge for organic and elemental carbon (OC/EC) analysis. Each cartridge contains a sharp-cut $\mathrm{PM}_{2.5}$ impactor operating at a flow rate of $10 \mathrm{~L} / \mathrm{min}$ (Nylon and Quartz) or $16.7 \mathrm{~L} / \mathrm{min}$ (Teflon). The samplers recorded and stored the sampling time and flow rate for each of the events. In addition, the temperature and relative humidity were recorded continuously. All sampling cartridges were prepared and assembled in the laboratory for each sampling event. The collected PM samples (filters) were transported to the laboratory in a cooler with ice packs $\left(\right.$ at $4^{\circ} \mathrm{C}$ ) for analysis. The filed sampling campaign covered four seasons from December 2008-December 2009. In total, 312 samples were taken across winter, spring, summer and fall seasons.

\section{2. $\mathrm{PM}_{2.5}$ Chemical Speciation Analysis}

Analyses of the $\mathrm{PM}_{2.5}$ samples were conducted at RTI International (Research Triangle Park, North Carolina). The analytical methods followed the standard operation procedures (SOPs) for the US EPA National PM $_{2.5}$ Chemical Speciation Network [15]. Table 1 lists all the chemical species that were analyzed for the study. 


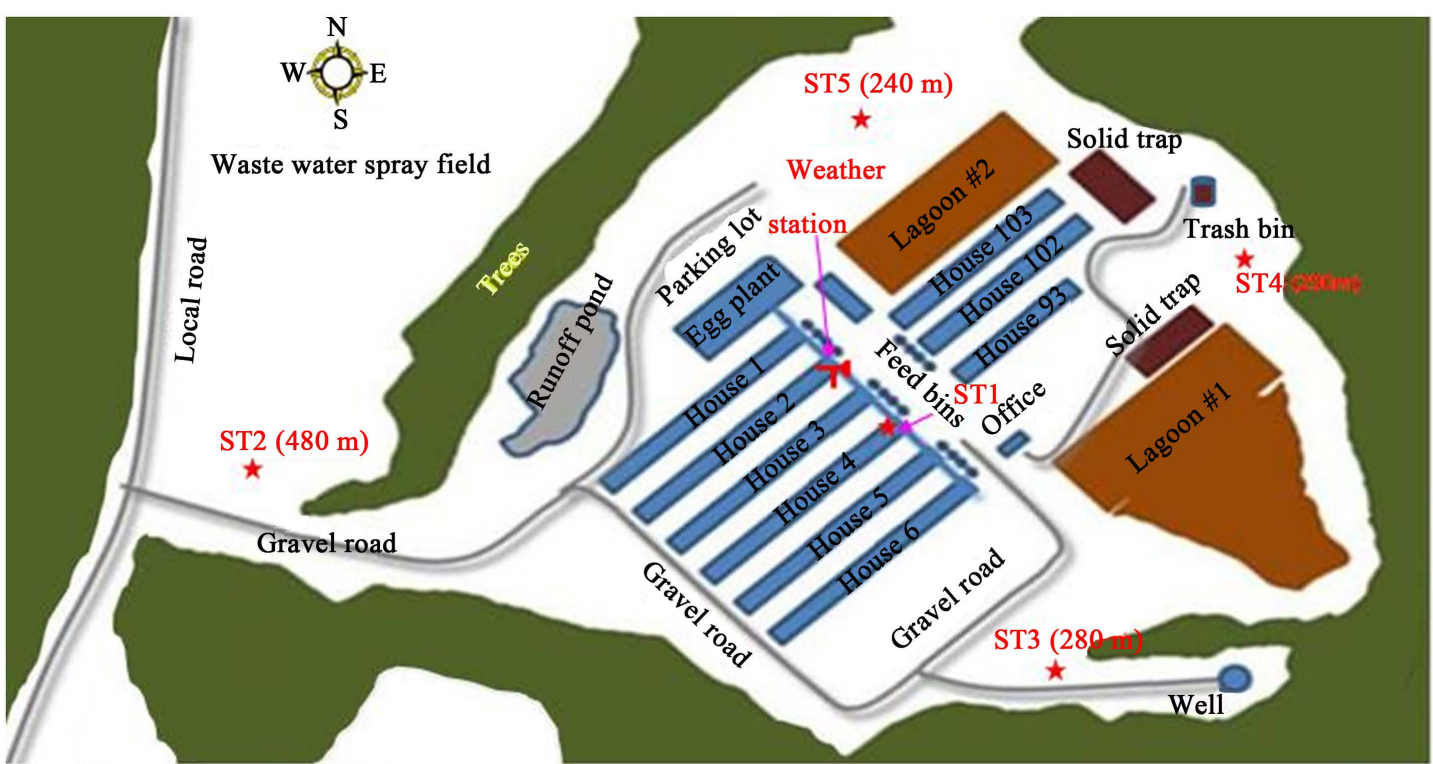

Figure 1. The egg production farm layout and the $\mathrm{PM}_{2.5}$ sampling stations [14].

Table 1. Summary of chemical species analyzed for the study.

\begin{tabular}{ccc}
\hline Filter type & Chemical species & Analytical method \\
\hline & & $\mathrm{PM}_{2.5}$ mass \\
PTFE & $\mathrm{Ag}, \mathrm{Al}, \mathrm{As}, \mathrm{Ba}, \mathrm{Br}, \mathrm{Ca}, \mathrm{Cd}, \mathrm{Ce}, \mathrm{Cl}, \mathrm{Co}, \mathrm{Cr}, \mathrm{Cs}, \mathrm{Cu}, \mathrm{Fe}, \mathrm{In}, \mathrm{Gravimetric}$ \\
& $\mathrm{K}, \mathrm{Mg}, \mathrm{Mn}, \mathrm{Na}, \mathrm{Ni}, \mathrm{P}, \mathrm{Pb}, \mathrm{Rb} \mathrm{S}, \mathrm{Sb}, \mathrm{Se}, \mathrm{Si}, \mathrm{Sn}, \mathrm{Sr}, \mathrm{Ti}, \mathrm{V}, \mathrm{Zn}, \mathrm{Zr}$ & Trace elements by X-ray fluorescence \\
Nylon & $\mathrm{Cl}^{-}, \mathrm{K}^{+}, \mathrm{Na}^{+}, \mathrm{NO}_{4}^{+}, \mathrm{NO}_{3}^{-}, \mathrm{SO}_{4}^{2-}$ & Anions and cations by IC \\
Quartz & $\mathrm{EC}, \mathrm{OC}$ & Thermal/optical-transmittance carbon analyzer \\
\hline
\end{tabular}

Gravimetric analyses of $\mathrm{PM}_{2.5}$ samples were conducted in an environmentally-controlled filter weighing chamber at RTI. This chamber's temperature was controlled between $20^{\circ} \mathrm{C}-23^{\circ} \mathrm{C}$ with a standard deviation less than $2^{\circ} \mathrm{C}$ and 24-hour mean RH between 30\% - 40\% with a standard deviation less than 5\% (RTI International, 2003). The filters (Whatman $2 \mu \mathrm{m}$ PTFE $46.2 \mathrm{~mm}$ ) were conditioned in this chamber for a minimum of 48 hours before the pre and post weights of the filter were taken by a microbalance with minimum readability of $\pm 1 \mu \mathrm{g}$ and a repeatability of $1 \mu \mathrm{g}$.

The energy dispersive X-ray fluorescence (EDXRF) spectrometry was applied to determine trace elements of $\mathrm{PM}_{2.5}$ deposits on the Teflon filters. The EDXRF technique is capable of analyzing elements with atomic number 11 (sodium) through 92 (uranium). The 33 elements analyzed by the EDXRF technique for this project.

Nylon filters were extracted for anion and cations analysis by ion chromatography (IC). In this process, $\mathrm{NO}_{4}^{+}, \mathrm{Na}^{+}$, and $\mathrm{K}^{+}$were analyzed by the ICwitha chemical suppressor, and the eluent was $11 \mathrm{mmol} / \mathrm{L}$ sulfuric acid $\left(\mathrm{H}_{2} \mathrm{SO}_{4}\right)$ at a flow rate of $1 \mathrm{~mL} / \mathrm{min}$. $\mathrm{SO}_{4}^{2-}, \mathrm{Cl}^{-}$, and $\mathrm{NO}_{3}^{-}$were analyzed by the IC with another chemical suppressor, and the eluent was $0.3 \mathrm{mmol} / \mathrm{L} \mathrm{NaHCO}_{3} / 2.7 \mathrm{mmol} / \mathrm{L} \mathrm{Na} \mathrm{CO}_{3}$ at a flow rate of $1.0 \mathrm{~mL} / \mathrm{min}$ [15].

Organic carbon and EC were analyzed by the thermo-optical carbon analyzer, which was based on the preferential oxidation of OC/EC at different temperature. Organic carbon can be volatilized from quartz filter in a helium at low temperature, while EC cannot be volatilized at helium and low temperature, then oxygen $\left(\mathrm{O}_{2}\right)$ was introduced to oxidized EC. All OC/EC were oxidized to $\mathrm{CO}_{2}$ and then reduction to methane $\left(\mathrm{CH}_{4}\right)$, then methane $\left(\mathrm{CH}_{4}\right)$ was quantified by flame ionization detector (FID). Optical method was used to correct pyrolysis OC by continuously monitoring the filter reflectance or transmittance. This method reports only carbon contents and does not directly account for the mass of hydrogen, oxygen and any other elements.

\subsection{Censored Data Analysis}

The EDXRF is a great method for analyzing trace elements in a large numbers of filters with high throughput. 
However, for normally loaded filters, certain elements are usually below detection limit (DL). When the level of an element in a sample is less than the DLs, the XRF automatically generates censored data, and reports as zero mass on filter. The retention of values less than DL in data sets can avoid unnecessary loss of information. An obvious question then is how to estimate the mean and standard deviation (SD) of the population from which the sample was drawn. Generally, deletion or substitution methods provide poor estimates of the mean and variance of censored samples. These deletion and substitution methods include the use of values above the DL only, or substitution of 0 , DL/2 or the DL for the below DL values during the calculation of mean and variance. A variety of statistical methods provide better estimators. The approach used in this study for processing measured elements concentrations was: 1) the censored values were replaced by DLs; 2) parametric method was applied to assume a normal distribution and maximum likelihood method was used to estimate parameters, based primarily on the non-censored data but incorporating the size of the censored and non-censored components of the sample.

\section{Results and Discussion}

\section{1. $\mathrm{PM}_{2.5}$ Elemental Compositions}

The values below the DL were a major statistical complication. The use of such data (left-censored) for detecting trends or relationship can be compromised despite the most rigorous quality assurance program. Figure 2 shows two sample data sets for element V (Vanadium) from ambient stations (ST4 and ST5). Both distributions were high skewed to right because of data truncation. The percentages of zero values for $\mathrm{V}$ were as high as $58 \%$ and $62 \%$ for ST4 and ST5, respectively.

Table 2 lists the summary of the reported zero value percentages at ST1 to ST5 (exactly percentage values shown in Tables 3-7). Using ST4 as an example, Ag, Ba, Cd, Ce, Co, Cs, In, Mg, P, Rb, Sn, Sr and Zr had more than $66.7 \%$ reported zero values, $\mathrm{Al}, \mathrm{Ni}, \mathrm{Pb}, \mathrm{Sb}, \mathrm{V}$ had reported zero values in the range of 33.3 to $66.7 \%$, and As, Br, Ca, Cl, Cr, Cu, Fe, K, Mn, Na, S, Se, Si, Ti and Zn had low percentage zero value (<33.3\%). Obviously, direct substitution or deletion of zero values will provide poor estimates. These substitution and deletion methods include the use of values above the DL only, or substitution of 0 , with DL/2, or the DL for below DL

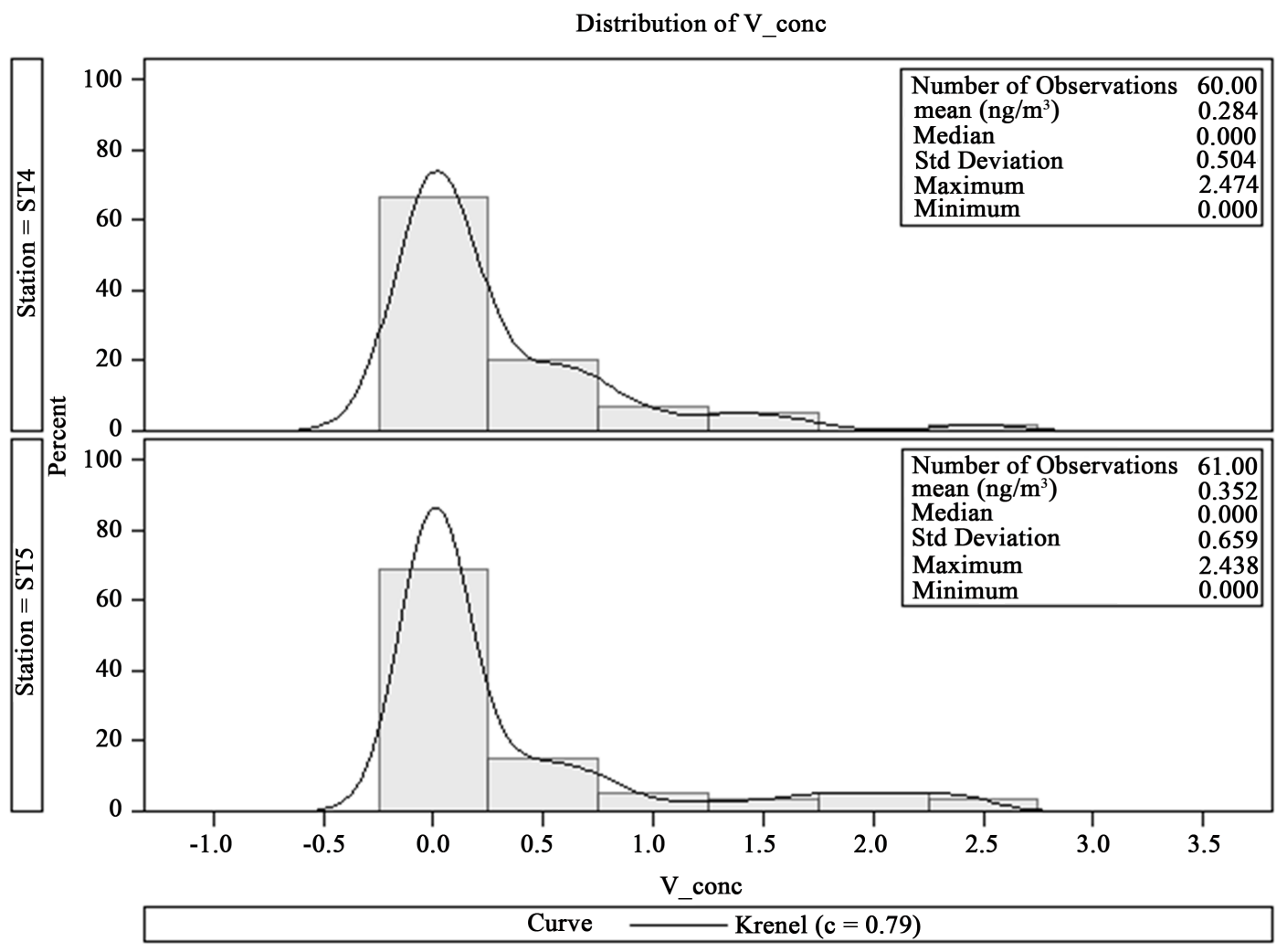

Figure 2. Left censored data for V from ambient stations: ST4 -Up and ST5-down. 
Table 2. The reported zero value percentages in samples taken at ST1 to ST5.

\begin{tabular}{|c|c|c|c|}
\hline $\begin{array}{c}\text { Zero values } \\
\text { percentage (\%) }\end{array}$ & $>66.7 \%$ & $33.3 \%$ to $66.7 \%$ & $<33.3 \%$ \\
\hline ST1 & Ag, Cd, Cr, Cs, In, Sb, Sn, Zr & $\mathrm{Al}, \mathrm{Ba}, \mathrm{Ce}, \mathrm{Co}, \mathrm{Rb}, \mathrm{Sr}$ & $\begin{array}{c}\text { As, } \mathrm{Br}, \mathrm{Ca}, \mathrm{Cl}, \mathrm{Cu}, \mathrm{Fe}, \mathrm{K}, \mathrm{Mg}, \mathrm{Mn}, \mathrm{Na} \text {, } \\
\text { Ni, P, Pb, S, Se, Si, Ti, V, Zn }\end{array}$ \\
\hline ST2 & Ba, Cd, Ce, Co, Cs, In, Mg, P, Rb, Sn, Sr, Zr & $\mathrm{Cr}, \mathrm{Ni}, \mathrm{Pb}, \mathrm{Sb}, \mathrm{V}$ & $\begin{array}{l}\mathrm{Al}, \mathrm{As}, \mathrm{Br}, \mathrm{Ca}, \mathrm{Cl}, \mathrm{Cu}, \mathrm{Fe}, \mathrm{K} \\
\text { Mn, Na, S, Se, Si, Ti, Zn }\end{array}$ \\
\hline ST3 & Ag, Ba, Ce, Cs, In, Mg, P, Rb, Sn, Sr, Zr & Al, Cd, Co, Ni, Pb, Sb, V & $\begin{array}{l}\text { As, Br, Ca, Cl, Cr, Cu, Fe, K, } \\
\text { Mn, Na, S, Se, Si, Ti, Zn }\end{array}$ \\
\hline ST4 & Ag, Ba, Cd, Ce, Co, Cs, In, Mg, P, Rb, Sn, Sr, Zr & $\mathrm{Al}, \mathrm{Ni}, \mathrm{Pb}, \mathrm{Sb}, \mathrm{V}$ & $\begin{array}{l}\text { As, Br, Ca, Cl, Cr, Cu, Fe, K, } \\
\text { Mn, Na, S, Se, Si, Ti, Zn }\end{array}$ \\
\hline ST5 & Ba, Cd, Ce, Co, Cs, In, Mg, P, Rb, Sb, Sn, Sr, Zr & $\mathrm{Al}, \mathrm{As}, \mathrm{Cr}, \mathrm{Ni}, \mathrm{V}$ & $\begin{array}{l}\text { Ag, Br, Ca, Cl, Cu, Fe, K, Mn, } \\
\text { Na, Pb, S, Se, Si, Ti, Zn }\end{array}$ \\
\hline
\end{tabular}

Table 3. ST1 element concentration $\left(\mathrm{ng} / \mathrm{m}^{3}\right)$ : different method comparison.

\begin{tabular}{|c|c|c|c|c|c|c|c|c|c|c|c|}
\hline Element & $\begin{array}{c}\text { Mean } \\
\text { W/O zero }\end{array}$ & $\begin{array}{c}\mathrm{SD}^{\mathrm{a}} \\
\mathrm{W} / \mathrm{O} \text { zero }\end{array}$ & $\begin{array}{c}\mathrm{W} / \mathrm{O} \\
\text { zero } \mathrm{N}_{\mathrm{Obs}}{ }^{\mathrm{b}}\end{array}$ & $\begin{array}{c}\text { Mean } \\
\text { W/zero }\end{array}$ & $\begin{array}{c}\text { SD } \\
\text { W/zero }\end{array}$ & $\begin{array}{l}\text { Uncensored } \\
\text { mean }\end{array}$ & $\begin{array}{l}\text { Uncensored } \\
\text { SD }\end{array}$ & $\begin{array}{c}\text { Censored } \\
\text { mean }\end{array}$ & $\begin{array}{c}\text { Censored } \\
\text { SD }\end{array}$ & $\begin{array}{l}\text { Total } \\
\mathrm{N}_{\text {Obs }}\end{array}$ & $\begin{array}{c}\text { Zero/ } \\
\text { total }\end{array}$ \\
\hline $\mathrm{Ag}$ & 3.62 & 1.44 & 9 & 0.56 & 1.43 & -6.00 & 0.50 & 0.51 & 0.08 & 58 & 84 \\
\hline $\mathrm{Al}$ & 45.15 & 47.78 & 31 & 24.13 & 41.45 & 0.07 & 16.51 & 25.96 & 7.72 & 58 & 47 \\
\hline As & 8.65 & 5.05 & 58 & 8.65 & 5.05 & 8.65 & 2.80 & 8.72 & 2.75 & 58 & 0.0 \\
\hline $\mathrm{Ba}$ & 17.33 & 12.23 & 30 & 8.96 & 12.35 & 3.77 & 15.19 & 8.95 & 11.00 & 58 & 48 \\
\hline $\mathrm{Br}$ & 4.68 & 3.22 & 58 & 4.68 & 3.22 & 4.68 & 2.14 & 4.76 & 2.07 & 58 & 0.0 \\
\hline $\mathrm{Ca}$ & 2991 & 3305 & 58 & 2991 & 33054 & 2991 & 3010 & 3187 & 2829 & 58 & 0.0 \\
\hline $\mathrm{Cd}$ & 3.45 & 1.86 & 10 & 0.59 & 1.51 & -5.35 & 0.21 & 0.56 & 0.04 & 58 & 83 \\
\hline $\mathrm{Ce}$ & 6.29 & 5.40 & 32 & 3.47 & 5.08 & 1.42 & 5.88 & 3.57 & 4.18 & 58 & 45 \\
\hline $\mathrm{Cl}$ & 1601 & 1597 & 58 & 1601 & 15979 & 1601 & 15647 & 1633 & 1532 & 58 & 0.0 \\
\hline Co & 0.63 & 0.48 & 30 & 0.32 & 0.47 & 0.09 & 0.47 & 0.33 & 0.30 & 58 & 48 \\
\hline $\mathrm{Cu}$ & 11.78 & 14.73 & 58 & 11.78 & 14.73 & 11.78 & 12.12 & 13.19 & 10.89 & 58 & 0.0 \\
\hline $\mathrm{Fe}$ & 185.66 & 113.12 & 58 & 185.66 & 113.12 & 185.66 & 100.90 & 185.98 & 100.59 & 58 & 0.0 \\
\hline In & 6.64 & 11.79 & 13 & 1.49 & 6.09 & -13.45 & 1.42 & 1.86 & 0.28 & 58 & 78 \\
\hline K & 18091 & 1716 & 58 & 18091 & 1716 & 18091 & 16860 & 1830 & 16652 & 58 & 0.0 \\
\hline $\mathrm{Mg}$ & 5312 & 5352 & 57 & 522 & 5358 & 5213 & 511 & 5406 & 493 & 58 & 1.7 \\
\hline $\mathrm{Mn}$ & 35.00 & 40.21 & 58 & 35.00 & 40.21 & 35.00 & 35.63 & 37.86 & 33.03 & 58 & 0.0 \\
\hline $\mathrm{Na}$ & 758.22 & 731.74 & 58 & 758.22 & 731.74 & 758.22 & 715.62 & 770.18 & 703.79 & 58 & 0.0 \\
\hline $\mathrm{Ni}$ & 0.77 & 0.60 & 49 & 0.65 & 0.62 & 0.61 & 0.54 & 0.66 & 0.49 & 58 & 16 \\
\hline $\mathrm{P}$ & 1262 & 1253 & 58 & 1262 & 1253 & 1262 & 1212 & 1294 & 1180 & 58 & 0.0 \\
\hline $\mathrm{Pb}$ & 2.72 & 2.72 & 45 & 2.11 & 2.65 & 1.64 & 1.11 & 2.26 & 0.82 & 58 & 22 \\
\hline $\mathrm{Rb}$ & 1.58 & 1.15 & 37 & 1.01 & 1.19 & 0.79 & 1.29 & 1.01 & 1.10 & 58 & 36 \\
\hline $\mathrm{S}$ & 1761 & 894 & 58 & 1761 & 894 & 1761 & 797 & 1761 & 796 & 58 & 0.0 \\
\hline $\mathrm{Sb}$ & 10.45 & 10.19 & 16 & 2.88 & 7.03 & -10.87 & 0.86 & 2.97 & 0.23 & 58 & 72 \\
\hline Se & 0.45 & 0.35 & 39 & 0.31 & 0.36 & 0.20 & 0.11 & 0.31 & 0.07 & 58 & 33 \\
\hline $\mathrm{Si}$ & 263.78 & 216.54 & 58 & 263.78 & 216.54 & 263.78 & 159.69 & 271.95 & 152.53 & 58 & 0.0 \\
\hline Sn & 5.13 & 4.76 & 14 & 1.24 & 3.17 & -6.48 & 2.18 & 1.26 & 0.46 & 58 & 76 \\
\hline $\mathrm{Sr}$ & 6.99 & 6.64 & 31 & 3.74 & 5.97 & 0.82 & 6.28 & 3.92 & 4.09 & 58 & 47 \\
\hline $\mathrm{Ti}$ & 5.11 & 4.27 & 55 & 4.85 & 4.31 & 4.73 & 1.12 & 5.06 & 0.98 & 58 & 5.2 \\
\hline $\mathrm{V}$ & 3.39 & 2.07 & 55 & 3.21 & 2.15 & 3.19 & 1.93 & 3.21 & 1.92 & 58 & 5.2 \\
\hline $\mathrm{Zn}$ & 255.46 & 236.11 & 58 & 255.46 & 236.11 & 255.46 & 230.00 & 258.58 & 226.91 & 58 & 0.0 \\
\hline $\mathrm{Zr}$ & 2.63 & 1.42 & 4 & 0.18 & 0.75 & -8.02 & 0.53 & 0.17 & 0.04 & 58 & 93 \\
\hline
\end{tabular}

${ }^{\mathrm{a}} \mathrm{SD}=$ standard deviation; ${ }^{\mathrm{b}}$ Nobs $=$ number of observations. 
Table 4. ST2 element concentration $\left(\mathrm{ng} / \mathrm{m}^{3}\right)$ : different method comparison.

\begin{tabular}{|c|c|c|c|c|c|c|c|c|c|c|c|}
\hline Element & $\begin{array}{c}\text { Mean } \\
\text { W/O zero }\end{array}$ & $\begin{array}{c}\text { SD } \\
\mathrm{W} / \mathrm{O} \text { zero }\end{array}$ & $\begin{array}{c}\mathrm{W} / \mathrm{O} \\
\text { zero } \mathrm{N}_{\text {Obs }}\end{array}$ & $\begin{array}{c}\text { Mean } \\
\text { W/zero }\end{array}$ & $\begin{array}{c}\text { SD } \\
\text { W/zero }\end{array}$ & $\begin{array}{l}\text { Uncensored } \\
\text { mean }\end{array}$ & $\begin{array}{l}\text { Uncensored } \\
\text { SD }\end{array}$ & $\begin{array}{c}\text { Censored } \\
\text { mean }\end{array}$ & $\begin{array}{l}\text { Censored } \\
\text { SD }\end{array}$ & $\begin{array}{l}\text { Total } \\
\mathrm{N}_{\text {Obs }}\end{array}$ & Zero/total \\
\hline $\mathrm{Ag}$ & 3.12 & 2.60 & 20 & 1.04 & 2.09 & -1.93 & 1.74 & 1.04 & 0.75 & 60 & 67 \\
\hline $\mathrm{Al}$ & 48.66 & 57.55 & 42 & 34.06 & 52.99 & 19.95 & 35.79 & 37.77 & 26.27 & 60 & 30 \\
\hline As & 0.85 & 0.46 & 41 & 0.58 & 0.55 & 0.44 & 0.21 & 0.56 & 0.16 & 60 & 32 \\
\hline $\mathrm{Br}$ & 2.81 & 1.59 & 60 & 2.81 & 1.59 & 2.81 & 0.48 & 2.83 & 0.47 & 60 & 0.0 \\
\hline $\mathrm{Ca}$ & 21.29 & 19.65 & 58 & 20.58 & 19.70 & 20.22 & 7.83 & 21.81 & 7.00 & 60 & 3.3 \\
\hline $\mathrm{Cd}$ & 3.19 & 3.07 & 16 & 0.85 & 2.10 & -3.51 & 2.10 & 0.88 & 0.74 & 60 & 73 \\
\hline $\mathrm{Cl}$ & 8.51 & 12.83 & 59 & 8.37 & 12.77 & 8.24 & 1.77 & 10.25 & 1.35 & 60 & 1.7 \\
\hline Co & 0.14 & 0.12 & 18 & 0.04 & 0.09 & -0.12 & 0.03 & 0.04 & 0.01 & 60 & 70 \\
\hline $\mathrm{Cr}$ & 0.69 & 0.49 & 39 & 0.45 & 0.52 & 0.28 & 0.03 & 0.45 & 0.02 & 60 & 35 \\
\hline Cs & 2.22 & 1.46 & 10 & 0.37 & 1.01 & -3.71 & 0.01 & 0.35 & 0.00 & 60 & 83 \\
\hline $\mathrm{Cu}$ & 1.20 & 2.48 & 48 & 0.96 & 2.27 & 0.59 & 0.01 & 1.36 & 0.00 & 60 & 20 \\
\hline $\mathrm{Fe}$ & 37.24 & 35.48 & 60 & 37.24 & 35.48 & 37.24 & 19.12 & 39.59 & 17.58 & 60 & 0.0 \\
\hline In & 5.11 & 5.40 & 14 & 1.19 & 3.34 & -6.93 & 1.00 & 1.25 & 0.25 & 60 & 77 \\
\hline K & 46.91 & 28.78 & 60 & 46.91 & 28.78 & 46.91 & 17.40 & 47.33 & 17.07 & 60 & 0.0 \\
\hline $\mathrm{Mg}$ & 6.62 & 5.36 & 9 & 0.99 & 3.09 & -12.93 & 2.22 & 0.98 & 0.29 & 60 & 85 \\
\hline Mn & 0.99 & 0.78 & 56 & 0.93 & 0.79 & 0.90 & 0.38 & 0.95 & 0.34 & 60 & 6.7 \\
\hline $\mathrm{Na}$ & 35.06 & 36.71 & 57 & 33.31 & 36.58 & 32.26 & 0.65 & 36.37 & 0.52 & 60 & 5.0 \\
\hline $\mathrm{Pb}$ & 1.60 & 2.26 & 34 & 0.91 & 1.87 & -0.07 & 0.28 & 1.07 & 0.13 & 60 & 43 \\
\hline $\mathrm{Rb}$ & 0.25 & 0.20 & 13 & 0.05 & 0.14 & -0.33 & 0.13 & 0.05 & 0.04 & 60 & 78 \\
\hline$S$ & 825.04 & 424.79 & 60 & 825.04 & 424.79 & 825.04 & 375.75 & 825.30 & 375.46 & 60 & 0.0 \\
\hline $\mathrm{Sb}$ & 10.15 & 10.07 & 21 & 3.55 & 7.63 & -5.90 & 3.86 & 3.70 & 1.60 & 60 & 65 \\
\hline Se & 0.57 & 0.39 & 43 & 0.41 & 0.42 & 0.32 & 0.18 & 0.41 & 0.15 & 60 & 28 \\
\hline $\mathrm{Si}$ & 129.77 & 137.76 & 60 & 129.77 & 137.76 & 129.77 & 75.42 & 141.12 & 68.06 & 60 & 0.0 \\
\hline Sn & 7.26 & 4.94 & 16 & 1.94 & 4.09 & -6.75 & 0.80 & 1.87 & 0.23 & 60 & 73 \\
\hline $\mathrm{Ti}$ & 3.61 & 3.91 & 54 & 3.25 & 3.87 & 3.02 & 2.13 & 3.55 & 1.82 & 60 & 10 \\
\hline $\mathrm{V}$ & 0.87 & 0.58 & 30 & 0.44 & 0.60 & 0.04 & 0.38 & 0.43 & 0.23 & 60 & 50 \\
\hline $\mathrm{Zn}$ & 4.10 & 2.52 & 60 & 4.10 & 2.52 & 4.10 & 0.87 & 4.15 & 0.84 & 60 & 0.0 \\
\hline $\mathrm{Zr}$ & 2.55 & 2.74 & 3 & 0.13 & 0.75 & -10.40 & 1.42 & 0.13 & 0.06 & 60 & 95 \\
\hline
\end{tabular}


Table 5. ST3 element concentration $\left(\mathrm{ng} / \mathrm{m}^{3}\right)$ : different method comparison.

\begin{tabular}{|c|c|c|c|c|c|c|c|c|c|c|c|}
\hline Element & $\begin{array}{c}\text { Mean W/O } \\
\text { zero }\end{array}$ & $\begin{array}{c}\text { SD } \\
\text { W/O zero }\end{array}$ & $\begin{array}{c}\mathrm{W} / \mathrm{O} \\
\text { zero } \mathrm{N}_{\text {Obs }}\end{array}$ & $\begin{array}{c}\text { Mean } \\
\text { W/zero }\end{array}$ & $\begin{array}{c}\text { SD } \\
\text { W/zero }\end{array}$ & $\begin{array}{l}\text { Uncensored } \\
\text { mean }\end{array}$ & $\begin{array}{c}\text { Uncensored } \\
\text { SD }\end{array}$ & $\begin{array}{c}\text { Censored } \\
\text { mean }\end{array}$ & $\begin{array}{c}\text { Censored } \\
\text { SD }\end{array}$ & $\begin{array}{l}\text { Total } \\
\mathrm{N}_{\text {Obs }}\end{array}$ & Zero/total \\
\hline $\mathrm{Ag}$ & 3.32 & 4.14 & 15 & 0.84 & 2.50 & -4.42 & 0.95 & 0.93 & 0.26 & 59 & 75 \\
\hline $\mathrm{Al}$ & 29.82 & 47.84 & 32 & 16.17 & 38.05 & -6.29 & 16.74 & 20.07 & 8.73 & 59 & 46 \\
\hline As & 0.58 & 0.44 & 44 & 0.43 & 0.46 & 0.34 & 0.04 & 0.44 & 0.03 & 59 & 25 \\
\hline $\mathrm{Br}$ & 2.68 & 1.69 & 59 & 2.68 & 1.69 & 2.68 & 0.84 & 2.72 & 0.82 & 59 & 0.0 \\
\hline $\mathrm{Ca}$ & 16.15 & 10.59 & 59 & 16.15 & 10.59 & 16.15 & 3.07 & 16.42 & 2.93 & 59 & 0.0 \\
\hline Cd & 4.65 & 4.61 & 22 & 1.73 & 3.58 & -2.53 & 2.43 & 1.81 & 1.06 & 59 & 63 \\
\hline $\mathrm{Cl}$ & 12.17 & 18.93 & 58 & 11.96 & 18.83 & 11.77 & 1.75 & 14.84 & 1.31 & 59 & 1.7 \\
\hline Co & 0.29 & 0.37 & 23 & 0.11 & 0.27 & -0.19 & 0.05 & 0.13 & 0.02 & 59 & 61 \\
\hline $\mathrm{Cr}$ & 0.68 & 0.70 & 44 & 0.50 & 0.67 & 0.37 & 0.09 & 0.54 & 0.06 & 59 & 25 \\
\hline Cs & 1.42 & 0.69 & 11 & 0.27 & 0.63 & -1.99 & 0.38 & 0.25 & 0.07 & 59 & 81 \\
\hline $\mathrm{Cu}$ & 1.39 & 3.74 & 44 & 1.04 & 3.28 & 0.26 & 0.27 & 1.70 & 0.14 & 59 & 25 \\
\hline $\mathrm{Fe}$ & 24.04 & 26.27 & 59 & 24.04 & 26.27 & 24.04 & 7.43 & 26.52 & 6.39 & 59 & 0.0 \\
\hline In & 4.22 & 6.24 & 15 & 1.07 & 3.58 & -6.47 & 0.41 & 1.25 & 0.10 & 59 & 75 \\
\hline $\mathrm{K}$ & 39.73 & 24.81 & 59 & 39.73 & 24.81 & 39.73 & 12.41 & 40.19 & 12.06 & 59 & 0.0 \\
\hline Mg & 2.26 & 2.27 & 11 & 0.42 & 1.29 & -3.87 & 0.45 & 0.43 & 0.08 & 59 & 81 \\
\hline Mn & 1.35 & 1.94 & 51 & 1.17 & 1.86 & 0.99 & 0.29 & 1.40 & 0.20 & 59 & 14 \\
\hline $\mathrm{Na}$ & 31.22 & 30.37 & 57 & 30.16 & 30.38 & 29.59 & 2.05 & 32.39 & 1.72 & 59 & 3.4 \\
\hline $\mathrm{Ni}$ & 0.29 & 0.26 & 29 & 0.14 & 0.23 & -0.02 & 0.01 & 0.15 & 0.00 & 59 & 51 \\
\hline $\mathrm{Pb}$ & 2.07 & 3.88 & 36 & 1.27 & 3.18 & -0.12 & 0.31 & 1.69 & 0.16 & 59 & 39 \\
\hline $\mathrm{Rb}$ & 0.36 & 0.52 & 10 & 0.06 & 0.24 & -0.84 & 0.22 & 0.07 & 0.04 & 59 & 83 \\
\hline S & 786.95 & 418.21 & 59 & 786.95 & 418.21 & 786.95 & 366.57 & 787.55 & 365.84 & 59 & 0.0 \\
\hline $\mathrm{Sb}$ & 8.71 & 9.96 & 21 & 3.10 & 7.21 & -6.47 & 2.47 & 3.36 & 0.91 & 59 & 64 \\
\hline Se & 0.48 & 0.34 & 42 & 0.34 & 0.36 & 0.26 & 0.14 & 0.34 & 0.11 & 59 & 29 \\
\hline $\mathrm{Si}$ & 75.81 & 94.61 & 58 & 74.53 & 94.31 & 73.58 & 26.42 & 85.35 & 21.74 & 59 & 1.7 \\
\hline Sn & 4.62 & 3.83 & 16 & 1.25 & 2.85 & -4.77 & 2.81 & 1.26 & 0.62 & 59 & 73 \\
\hline $\mathrm{Sr}$ & 1.57 & 0.17 & 2 & 0.05 & 0.29 & -6.28 & 0.94 & 0.05 & 0.05 & 59 & 97 \\
\hline $\mathrm{Ti}$ & 2.74 & 4.53 & 49 & 2.27 & 4.25 & 1.67 & 1.64 & 2.88 & 1.15 & 59 & 17 \\
\hline $\mathrm{Zn}$ & 4.21 & 2.45 & 59 & 4.21 & 2.45 & 4.21 & 0.91 & 4.25 & 0.89 & 59 & 0.0 \\
\hline
\end{tabular}


Table 6. ST4 element concentration $\left(\mathrm{ng} / \mathrm{m}^{3}\right)$ : different method comparison.

\begin{tabular}{|c|c|c|c|c|c|c|c|c|c|c|c|}
\hline Element & $\begin{array}{c}\text { Mean } \\
\text { W/O zero }\end{array}$ & $\begin{array}{c}\text { SD W/O } \\
\text { zero }\end{array}$ & $\begin{array}{c}\mathrm{W} / \mathrm{O} \\
\text { zero } \mathrm{N}_{\text {Obs }}\end{array}$ & $\begin{array}{c}\text { Mean } \\
\text { W/zero }\end{array}$ & $\begin{array}{c}\text { SD } \\
\text { W/zero }\end{array}$ & $\begin{array}{c}\text { Uncensored } \\
\text { mean }\end{array}$ & $\begin{array}{l}\text { Uncensored } \\
\text { SD }\end{array}$ & $\begin{array}{c}\text { Censored } \\
\text { mean }\end{array}$ & $\begin{array}{c}\text { Censored } \\
\text { SD }\end{array}$ & $\begin{array}{l}\text { Total } \\
\mathrm{N}_{\text {Obs }}\end{array}$ & Zero/total \\
\hline $\mathrm{Ag}$ & 3.76 & 3.67 & 15 & 0.94 & 2.43 & -4.43 & 1.27 & 0.97 & 0.36 & 60 & 75 \\
\hline $\mathrm{Al}$ & 28.92 & 51.11 & 28 & 13.50 & 37.51 & -17.17 & 22.07 & 17.38 & 10.56 & 60 & 53 \\
\hline As & 0.67 & 0.54 & 40 & 0.45 & 0.54 & 0.29 & 0.30 & 0.45 & 0.22 & 60 & 33 \\
\hline $\mathrm{Ba}$ & . & . & 0 & 0.00 & 0.00 & -0.55 & 0.04 & 0.00 & 0.00 & 60 & 100 \\
\hline $\mathrm{Br}$ & 2.62 & 1.73 & 59 & 2.57 & 1.75 & 2.56 & 0.80 & 2.61 & 0.76 & 60 & 1.7 \\
\hline $\mathrm{Ca}$ & 16.31 & 13.55 & 59 & 16.04 & 13.60 & 15.93 & 6.40 & 16.69 & 5.93 & 60 & 1.7 \\
\hline Cd & 3.22 & 2.28 & 15 & 0.80 & 1.79 & -3.26 & 0.96 & 0.78 & 0.28 & 60 & 75 \\
\hline $\mathrm{Cl}$ & 14.65 & 30.58 & 59 & 14.41 & 30.38 & 14.12 & 9.36 & 20.44 & 6.93 & 60 & 1.7 \\
\hline Co & 0.14 & 0.12 & 18 & 0.04 & 0.09 & -0.11 & 0.02 & 0.04 & 0.01 & 60 & 70 \\
\hline $\mathrm{Cr}$ & 0.54 & 0.32 & 41 & 0.37 & 0.37 & 0.27 & 0.00 & 0.36 & 0.00 & 60 & 32 \\
\hline Cs & 2.00 & 1.49 & 13 & 0.43 & 1.07 & -2.60 & 0.05 & 0.42 & 0.01 & 60 & 78 \\
\hline $\mathrm{Cu}$ & 1.13 & 2.67 & 45 & 0.84 & 2.36 & 0.31 & 0.01 & 1.28 & 0.01 & 60 & 25 \\
\hline $\mathrm{Fe}$ & 21.67 & 26.37 & 60 & 21.67 & 26.37 & 21.67 & 8.91 & 24.61 & 7.55 & 60 & 0.0 \\
\hline In & 3.37 & 2.34 & 12 & 0.67 & 1.69 & -4.68 & 0.24 & 0.65 & 0.05 & 60 & 80 \\
\hline $\mathrm{K}$ & 35.67 & 21.52 & 60 & 35.67 & 21.52 & 35.67 & 11.09 & 36.00 & 10.84 & 60 & 0.0 \\
\hline $\mathrm{Mg}$ & 6.20 & 3.04 & 8 & 0.83 & 2.37 & -11.87 & 1.94 & 0.76 & 0.32 & 60 & 87 \\
\hline $\mathrm{Mn}$ & 0.82 & 0.59 & 49 & 0.67 & 0.62 & 0.60 & 0.29 & 0.68 & 0.25 & 60 & 18 \\
\hline $\mathrm{Na}$ & 30.54 & 29.39 & 54 & 27.48 & 29.34 & 25.70 & 0.99 & 29.40 & 0.79 & 60 & 10 \\
\hline $\mathrm{Ni}$ & 0.17 & 0.18 & 32 & 0.09 & 0.16 & -0.00 & 0.04 & 0.10 & 0.02 & 60 & 47 \\
\hline $\mathrm{P}$ & 2.17 & 2.72 & 2 & 0.07 & 0.53 & -8.79 & 2.53 & 0.07 & 0.20 & 60 & 97 \\
\hline $\mathrm{Pb}$ & 1.67 & 2.49 & 34 & 0.95 & 2.04 & -0.12 & 0.20 & 1.14 & 0.10 & 60 & 43 \\
\hline $\mathrm{Rb}$ & 0.35 & 0.37 & 11 & 0.06 & 0.21 & -0.62 & 0.12 & 0.07 & 0.03 & 60 & 82 \\
\hline S & 776.88 & 409.87 & 60 & 776.88 & 409.87 & 776.88 & 358.48 & 777.33 & 357.96 & 60 & 0.0 \\
\hline $\mathrm{Sb}$ & 9.06 & 6.24 & 23 & 3.47 & 5.85 & -3.69 & 4.52 & 3.39 & 1.45 & 60 & 62 \\
\hline Se & 0.49 & 0.37 & 41 & 0.33 & 0.38 & 0.23 & 0.11 & 0.34 & 0.08 & 60 & 32 \\
\hline $\mathrm{Si}$ & 59.81 & 90.16 & 60 & 59.81 & 90.16 & 59.81 & 29.30 & 73.13 & 23.60 & 60 & 0.0 \\
\hline Sn & 4.06 & 3.21 & 13 & 0.88 & 2.22 & -5.19 & 1.72 & 0.86 & 0.47 & 60 & 78 \\
\hline $\mathrm{Sr}$ & 2.44 & 2.44 & 6 & 0.24 & 1.02 & -6.85 & 1.27 & 0.25 & 0.16 & 60 & 90 \\
\hline $\mathrm{Ti}$ & 2.98 & 3.73 & 42 & 2.09 & 3.40 & 1.16 & 1.61 & 2.37 & 1.10 & 60 & 30 \\
\hline $\mathrm{V}$ & 0.68 & 0.58 & 25 & 0.28 & 0.50 & -0.19 & 0.40 & 0.29 & 0.21 & 60 & 58 \\
\hline $\mathrm{Zn}$ & 3.72 & 2.24 & 60 & 3.72 & 2.24 & 3.72 & 1.04 & 3.76 & 1.01 & 60 & 0.0 \\
\hline
\end{tabular}


Table 7. ST5 element concentration $\left(\mathrm{ng} / \mathrm{m}^{3}\right)$ : different method comparison.

\begin{tabular}{|c|c|c|c|c|c|c|c|c|c|c|c|}
\hline Element & $\begin{array}{c}\text { Mean } \\
\text { W/O zero }\end{array}$ & $\begin{array}{l}\text { SD W/O } \\
\text { zero }\end{array}$ & $\begin{array}{c}\mathrm{W} / \mathrm{O} \\
\text { zero } \mathrm{N}_{\text {Obs }}\end{array}$ & $\begin{array}{c}\text { Mean } \\
\text { W/zero }\end{array}$ & $\begin{array}{c}\text { SD } \\
\text { W/zero }\end{array}$ & $\begin{array}{c}\text { Uncensored } \\
\text { mean }\end{array}$ & $\begin{array}{l}\text { Uncensored } \\
\text { SD }\end{array}$ & $\begin{array}{l}\text { Censored } \\
\text { mean }\end{array}$ & $\begin{array}{c}\text { Censored } \\
\text { SD }\end{array}$ & $\begin{array}{l}\text { Total } \\
\mathrm{N}_{\text {Obs }}\end{array}$ & Zero/total \\
\hline Ag & 2.84 & 2.06 & 61 & 0.47 & 1.39 & -5.10 & 0.18 & 0.46 & 0.03 & 61 & 0.0 \\
\hline $\mathrm{Al}$ & 38.36 & 61.15 & 32 & 20.12 & 48.01 & -10.12 & 32.42 & 24.83 & 17.70 & 61 & 48 \\
\hline As & 0.61 & 0.54 & 39 & 0.39 & 0.52 & 0.21 & 0.27 & 0.40 & 0.19 & 61 & 36 \\
\hline $\mathrm{Ba}$ & 0.46 & . & 1 & 0.01 & 0.06 & -2.49 & 0.35 & 0.01 & 0.01 & 61 & 98 \\
\hline $\mathrm{Br}$ & 2.74 & 1.90 & 61 & 2.74 & 1.90 & 2.74 & 0.63 & 2.80 & 0.60 & 61 & 0.0 \\
\hline $\mathrm{Ca}$ & 18.68 & 16.79 & 60 & 18.37 & 16.82 & 18.22 & 9.50 & 19.30 & 8.77 & 61 & 1.6 \\
\hline $\mathrm{Cd}$ & 4.16 & 3.28 & 17 & 1.16 & 2.53 & -3.82 & 0.70 & 1.15 & 0.21 & 61 & 72 \\
\hline $\mathrm{Cl}$ & 12.90 & 25.74 & 60 & 12.69 & 25.58 & 12.40 & 4.29 & 17.61 & 3.07 & 61 & 1.6 \\
\hline Co & 0.27 & 0.23 & 16 & 0.07 & 0.17 & -0.29 & 0.02 & 0.07 & 0.01 & 61 & 74 \\
\hline $\mathrm{Cr}$ & 0.81 & 0.83 & 40 & 0.53 & 0.78 & 0.27 & 0.15 & 0.57 & 0.09 & 61 & 34 \\
\hline Cs & 1.09 & 1.20 & 11 & 0.20 & 0.65 & -1.86 & 0.55 & 0.21 & 0.13 & 61 & 82 \\
\hline $\mathrm{Cu}$ & 1.21 & 2.75 & 48 & 0.95 & 2.48 & 0.48 & 0.37 & 1.41 & 0.22 & 61 & 21 \\
\hline $\mathrm{Fe}$ & 26.70 & 32.90 & 61 & 26.70 & 32.90 & 26.70 & 13.96 & 30.39 & 11.97 & 61 & 0.0 \\
\hline In & 4.31 & 4.26 & 11 & 0.78 & 2.41 & -7.61 & 1.35 & 0.80 & 0.27 & 61 & 82 \\
\hline $\mathrm{K}$ & 40.87 & 28.18 & 61 & 40.87 & 28.18 & 40.87 & 16.93 & 41.54 & 16.41 & 61 & 0.0 \\
\hline Mg & 16.94 & 41.63 & 12 & 3.33 & 19.07 & -46.80 & 13.54 & 4.82 & 2.96 & 61 & 80 \\
\hline Mn & 1.03 & 0.97 & 54 & 0.91 & 0.97 & 0.85 & 0.47 & 0.97 & 0.41 & 61 & 11 \\
\hline $\mathrm{Na}$ & 39.74 & 41.55 & 56 & 36.49 & 41.28 & 34.43 & 5.62 & 39.79 & 4.52 & 61 & 8.2 \\
\hline $\mathrm{Ni}$ & 0.27 & 0.21 & 30 & 0.13 & 0.20 & -0.01 & 0.04 & 0.13 & 0.02 & 61 & 51 \\
\hline $\mathrm{P}$ & 21.73 & 32.79 & 4 & 1.42 & 9.12 & -81.16 & 27.04 & 1.58 & 2.98 & 61 & 93 \\
\hline $\mathrm{Pb}$ & 1.60 & 1.82 & 43 & 1.13 & 1.69 & 0.69 & 0.08 & 1.25 & 0.05 & 61 & 30 \\
\hline $\mathrm{Rb}$ & 0.29 & 0.18 & 11 & 0.05 & 0.13 & -0.43 & 0.05 & 0.05 & 0.01 & 61 & 82 \\
\hline $\mathrm{S}$ & 755.66 & 417.00 & 61 & 755.66 & 417.00 & 755.66 & 337.70 & 756.99 & 336.36 & 61 & 0.0 \\
\hline $\mathrm{Sb}$ & 7.52 & 6.59 & 17 & 2.10 & 4.81 & -6.68 & 3.51 & 2.11 & 1.21 & 61 & 72 \\
\hline Se & 0.54 & 0.40 & 45 & 0.40 & 0.42 & 0.31 & 0.06 & 0.40 & 0.05 & 61 & 26 \\
\hline $\mathrm{Si}$ & 81.62 & 115.20 & 60 & 80.29 & 114.71 & 79.01 & 49.94 & 95.52 & 41.21 & 61 & 1.6 \\
\hline Sn & 8.23 & 14.88 & 11 & 1.48 & 6.86 & -20.54 & 1.93 & 1.85 & 0.30 & 61 & 82 \\
\hline $\mathrm{Sr}$ & 1.37 & 0.90 & 9 & 0.20 & 0.59 & -2.56 & 0.09 & 0.19 & 0.01 & 61 & 85 \\
\hline $\mathrm{Ti}$ & 2.76 & 4.02 & 48 & 2.17 & 3.73 & 1.53 & 1.75 & 2.61 & 1.25 & 61 & 21 \\
\hline V & 0.93 & 0.78 & 23 & 0.35 & 0.66 & -0.40 & 0.37 & 0.35 & 0.16 & 61 & 62 \\
\hline $\mathrm{Zn}$ & 3.95 & 2.72 & 61 & 3.95 & 2.72 & 3.95 & 1.26 & 4.02 & 1.21 & 61 & 0.0 \\
\hline
\end{tabular}


values during the calculation of mean and variance. In the study, the trace element data were treated as leftcensored; the Tobit model [16] was applied, and $\mathrm{PM}_{2.5}$ mass concentration was treated as a covariate. The Tobit model is a parametric method, assumes a normal distribution and uses maximum likelihood methods to estimate parameters, based primarily on the non-censored data but incorporating the size of the censored and non-censored components of the sample.

Tables 3-7 list the calculated results based on different methods for data validation: direct with zero, direct without zero (treated as missing), uncensored (truncated) and left censored. Taking Ag in ST1 as an example, there were total 58 observations, but the numbers of the reported zero values was 49 . When treating zero as missing, the mean was $3.62 \pm 1.44 \mathrm{ng} / \mathrm{m}^{3}(\mathrm{~N}=9)$. When doing direct calculation with zero values, the mean was $0.56 \pm 1.43 \mathrm{ng} / \mathrm{m}^{3}(\mathrm{~N}=58)$. The truncated method gave mean $=-5.00 \pm 0.50 \mathrm{ng} / \mathrm{m}^{3}(\mathrm{~N}=58)$. The left-censored method gave mean $=0.51 \pm 0.08 \mathrm{ng} / \mathrm{m}^{3}(\mathrm{~N}=58)$. Figure 3 and Figure 4 compare the resultant concentrations computed by the different methods. When the percentages of the values below DL (the zero value percentage)

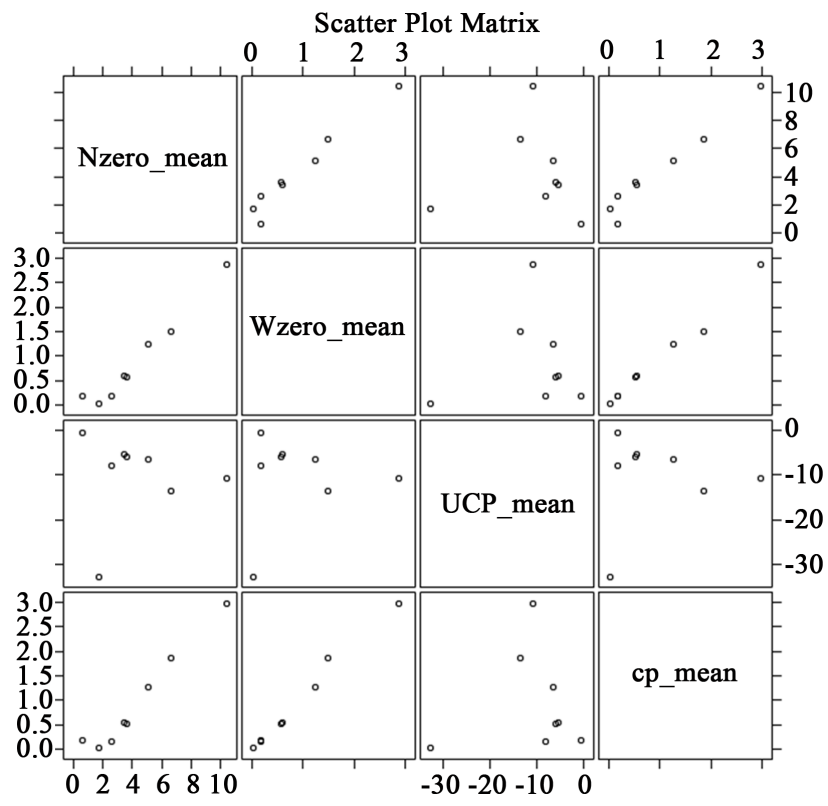

Scatter Plot Matrix

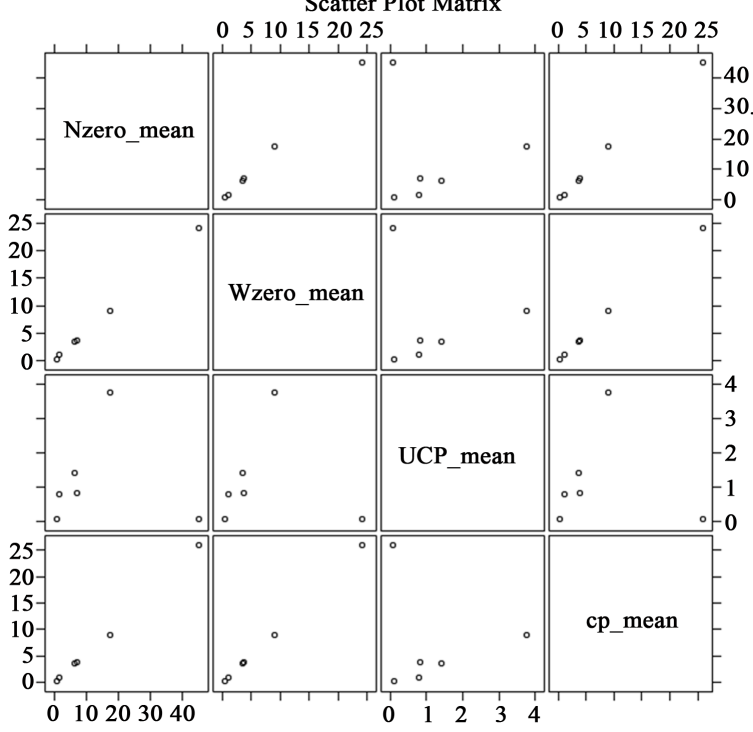

Scatter Plot Matrix

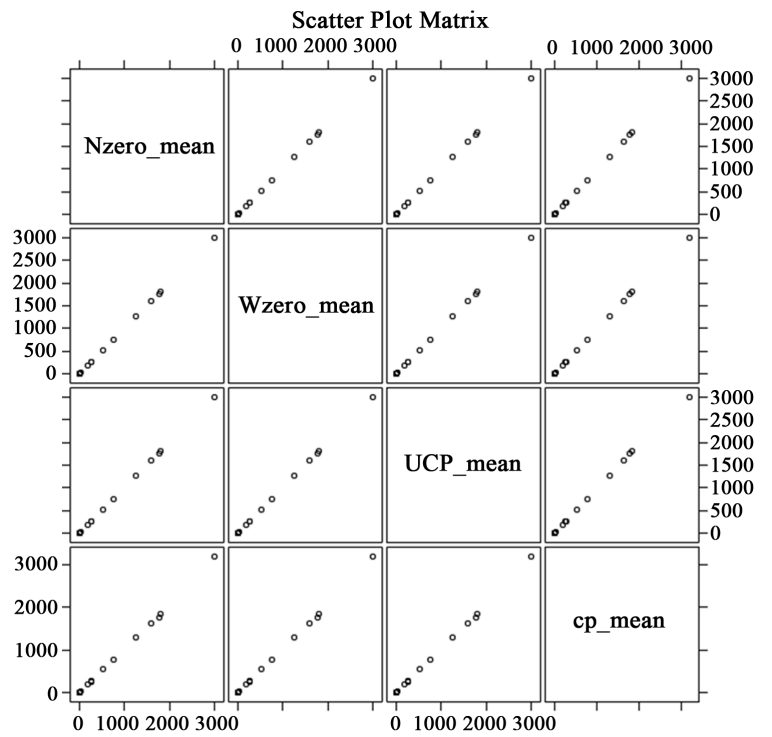

Figure 3. The different data analysis methods comparison for ST1: Above: zero value $\%>66.7 \%$, low left: $33.3 \%<$ zero value $\%<66.7 \%$, and low right: zero value $\%<33.3 \%$. 

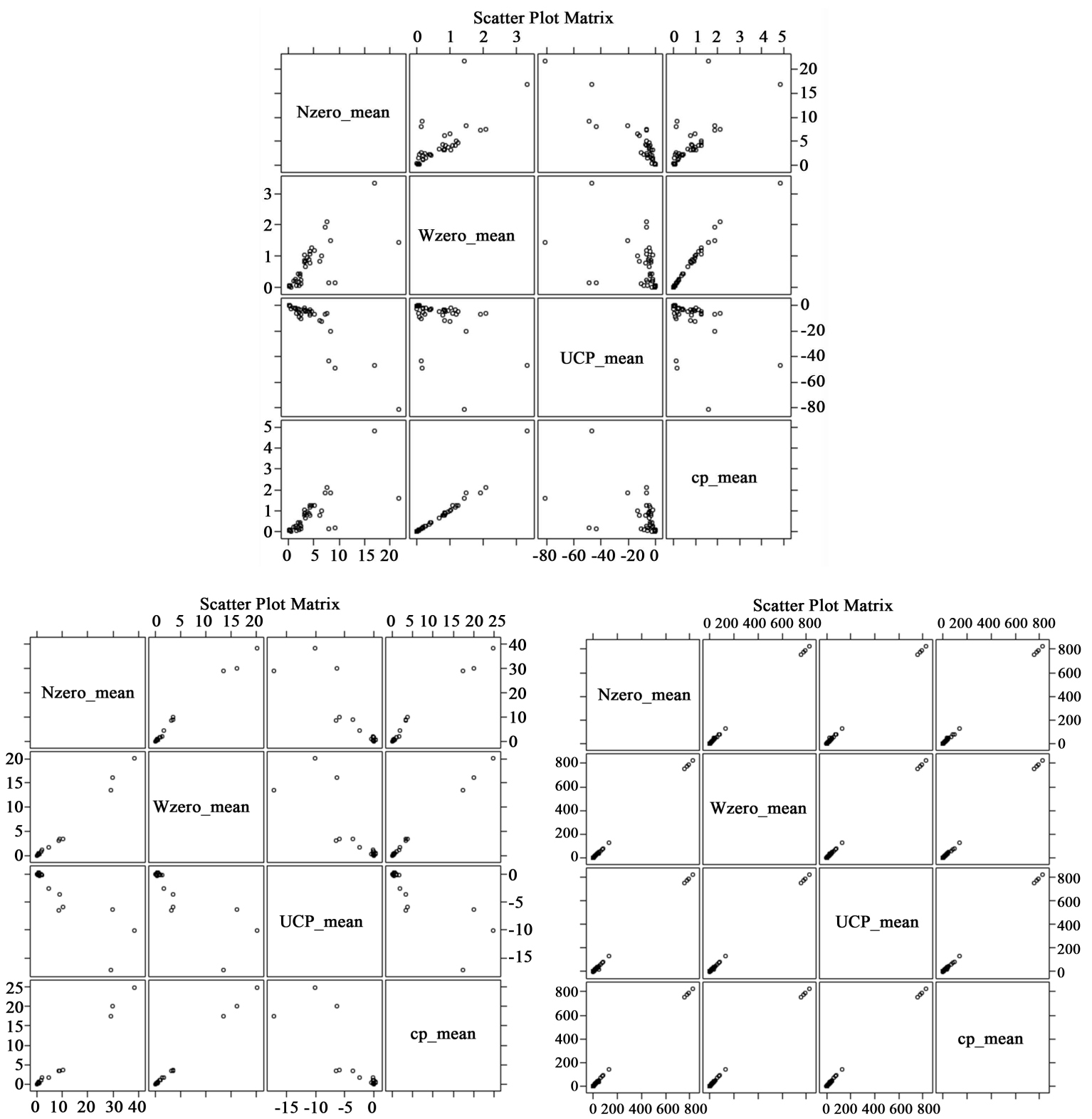

Figure 4. The different data analysis methods comparison for ambient stations (ST2-ST5): Above: zero value\% $>66.7 \%$, low left: $33.3 \%<$ zero value $\%<66.7 \%$, and low right: zero value $\%<33.3 \%$.

were below 66.7\%, all methods gave very constant results and did not show significant difference. However for the high percentages of the low DL values, different methods showed significant difference. The direct substitution and deletion methods gave biased results. The parametric methods can provide more effective and accurate estimator. However, it is obvious that the parametric methods are very sensitive to the choice of a distribution. Whether or not a distribution model is a good choice from a statistical point, there are many statistical tools that can help in decision making. After fitting a distribution model to a data set, diagnosing the model's fit or comparing the fit of different distributions are very important.

The measured elements were grouped based upon their properties as indicated in the period table (Figure 5). Following the group characterization, and treating the total measured elements as one unit, the pie charts for different sites were developed and are listed in Figure 6. For ST1 (in house), alkaline metals (Mg: $540 \pm 493$ $\mathrm{ng} / \mathrm{m}^{3}$, Ca: $3187 \pm 2829 \mathrm{ng} / \mathrm{m}^{3}$, Sr: $3.92 \pm 4.09 \mathrm{ng} / \mathrm{m}^{3}$, and Ba: $8.95 \pm 11.0 \mathrm{ng} / \mathrm{m}^{3}$ ) had 31.5\% of total measured element mass. Nonmetals (P: $1294 \pm 1180 \mathrm{ng} / \mathrm{m}^{3}$, S: $1761 \pm 796 \mathrm{ng} / \mathrm{m}^{3}$ ) had 25.8\% of total measured element 

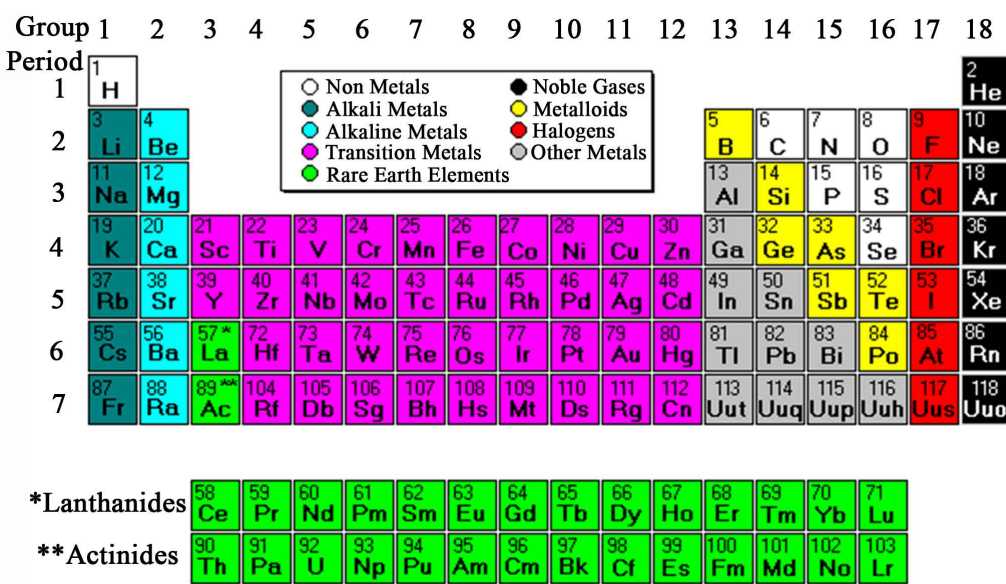

Figure 5. Periodic table and group label (“other metals” also known as "basic metals").
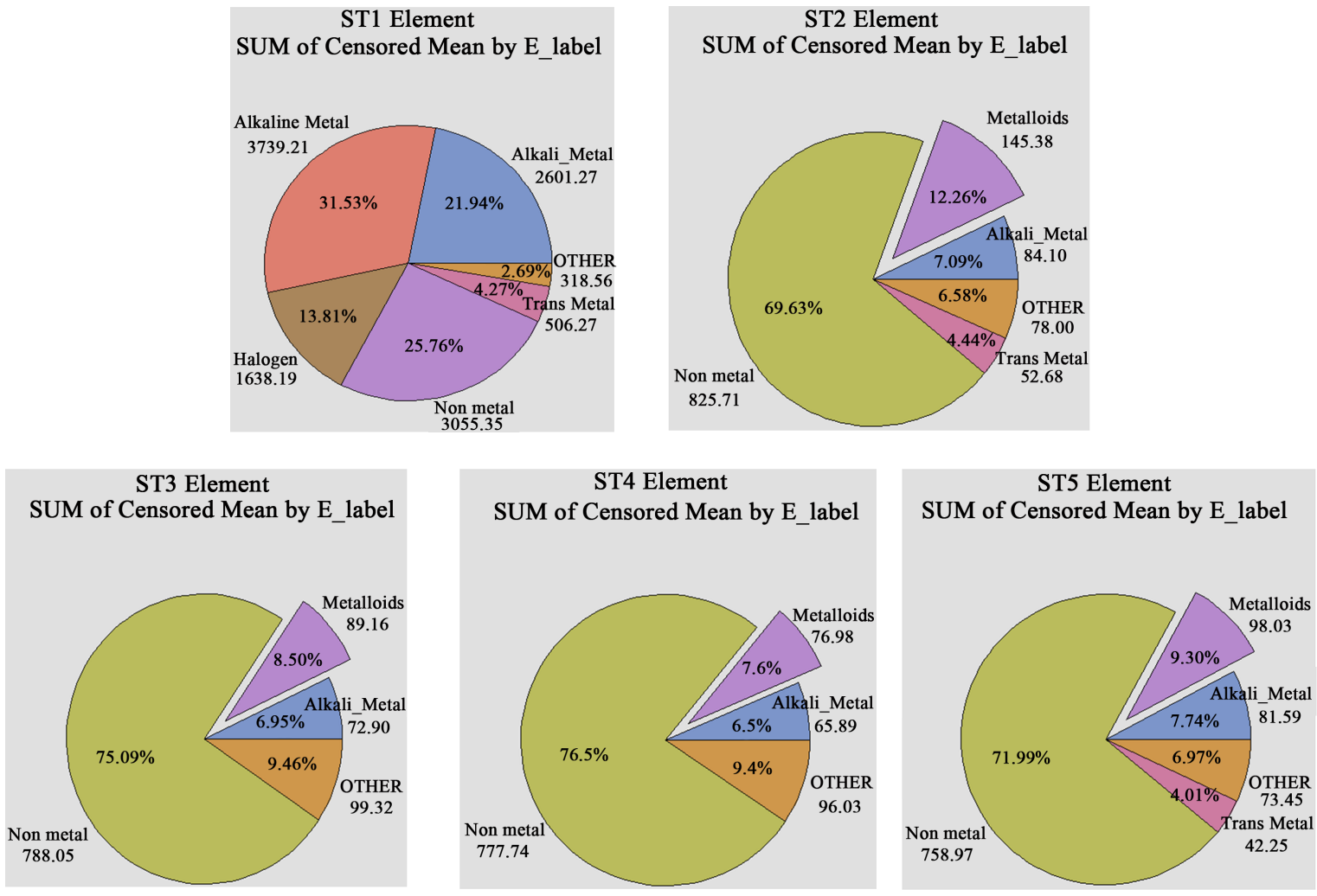

Figure 6. Elemental compositions of $\mathrm{PM}_{2.5}$ at different stations (ST1: in house, ST2-ST5: ambient).

mass, followed by alkali metals (Na: $770 \pm 704 \mathrm{ng} / \mathrm{m}^{3}, \mathrm{~K}: 1830 \pm 1665 \mathrm{ng} / \mathrm{m}^{3}$ ). For the ambient stations (ST2-ST5), the element compositions were different from ST1 (in house), the nonmetals had more than $70 \%$ of total measured elements (ST2: $825 \pm 375 \mathrm{ng} / \mathrm{m}^{3}$, ST3: $787 \pm 366 \mathrm{ng} / \mathrm{m}^{3}$, ST4: $77.3 \pm 358 \mathrm{ng} / \mathrm{m}^{3}$, ST5: $757 \pm 336 \mathrm{ng} / \mathrm{m}^{3}$ ). The majority sulfur (S) existed in particle phase as sulfate $\left(\mathrm{SO}_{4}^{2-}\right)$, which was an important component in $\mathrm{PM}_{2.5}$.

As shown in Figure 6, PM samples from Station 1 (ST1) and ambient stations (ST2-ST5) had big differences in elemental compositions, but for ambient stations (ST2-ST5), the elemental compositions were similar. For different elements, the statistical test results are shown in Table 8. For majority elements (such as: Al, As, Ba), the concentrations at ST1 were significantly different from ambient stations. Only for several elements (Ag, Cd, 
Table 8. Different elements at different locations.

\begin{tabular}{|c|c|c|c|}
\hline $\begin{array}{l}\text { House (ST1) vs. ambient (ST2-ST5) } \\
\text { (no sig diff) }\end{array}$ & House vs. ambient (sig diff) & Ambient (ST2-ST5) (no sig diff) & $\begin{array}{l}\text { Ambient (ST2-ST5) } \\
\text { (sig diff) }\end{array}$ \\
\hline Ag, Cd, Ce, Cs, In, Rb, Se, Sn, Zr & $\begin{array}{c}\text { Al, As, Ba, Br, Ca, Cl, Co, } \\
\mathrm{Cr}, \mathrm{Cu}, \mathrm{Fe}, \mathrm{K}, \mathrm{Mg}, \mathrm{Mn}, \mathrm{Na}, \mathrm{Ni} \\
\text { P, Pb, Rb, S, Si, Sr, Ti, V, Zn }\end{array}$ & $\begin{array}{l}\text { Ag, As, Ba, Br, Ca, Ce, Cl, } \\
\text { Co, Cr, Cs, Cu, In, K, Mg, Mn, } \\
\text { Na, Ni, P, Pb, Rb, S, Sb, Se, } \\
\text { Sn, Sr, Ti, V, Zn, Zr }\end{array}$ & $\mathrm{Al}, \mathrm{Cd}, \mathrm{Fe}, \mathrm{Si}$ \\
\hline
\end{tabular}

Ce, Cs, In, Rb, Se, Sn, and Zr), concentrations at ST1 and ambient stations (ST2-ST5) were very low, and showed no statistical difference. For ambient stations (ST2-ST5), Al, Fe, and Si at ST2 had much higher concentrations than ST3-ST5. All these elements (Al, Fe, and Si) are the marker elements from road dust emission source. This suggests the nearby local road and entrance grave road may have big impact on PM element compositions at ST2.

\section{2. $\mathrm{PM}_{2.5}$ Mass Balance Closure}

The average mass percentages of different chemical compositions in total $\mathrm{PM}_{2.5}$ masses are shown in Figure 7. The OC contributions were slightly different from ST1 to ST5, approximately 50\%. The ambient OC fractions were much higher than Pittsburgh area [17], which was only $23 \%$. The ions $\left(\mathrm{NH}_{4}^{+}, \mathrm{Na}^{+}, \mathrm{K}^{+}, \mathrm{SO}_{4}^{2-}, \mathrm{Cl}^{-}\right.$, and $\mathrm{NO}_{3}^{-}$) mass percentages at ST1-ST5 varied significantly. For ST1 (in-house station), all these ions accounted for less than $13 \%$ of the total $\mathrm{PM}_{2.5}$ mass. Specifically, $\mathrm{SO}_{4}^{2-}$ accounted for $4.8 \%$, and $\mathrm{NH}_{4}^{+}$accounted for $2.6 \%$. A considerable part of $\mathrm{PM}_{2.5}$ mass was organic in nature, instead of inorganic, and it was possibly originated from feed. Inside the production house, gas phase $\mathrm{NH}_{3}$ had relatively high concentrations (several-to-hundred $\mathrm{ppm})$, but acidic gases $\left(\mathrm{NO}_{\mathrm{x}}, \mathrm{SO}_{\mathrm{x}}\right.$ ) were unlikely in high concentrations to react with $\mathrm{NH}_{3}$ to form secondary inorganic aerosols, also the short residence time in the house was likely another reason for limited inorganic ammonium aerosol formation. It was also possible that $\mathrm{NH}_{3}$ may react with $\mathrm{PM}_{2.5}$ bounded moisture to form ammonium hydroxide $\left(\mathrm{NH}_{4} \mathrm{OH}\right)$. However, $\mathrm{NH}_{4} \mathrm{OHmost}$ likely evaporated during the filter transportation, storing and conditioning prior to IC analyses. The mass percentages of ions were similar at the 4 ambient stations, and $\mathrm{SO}_{4}^{2-}, \mathrm{NO}_{3}^{-}$and $\mathrm{NH}_{4}^{+}$were the dominate ion species on a mass basis (Figure 7). Estimates of the cumulative contributions of $\mathrm{SO}_{4}^{2-}, \mathrm{NO}_{3}^{-}$and $\mathrm{NH}_{4}^{+}$to total $\mathrm{PM}_{2.5}$ mass were $38 \%$ to $44 \%$, which were slightly lower than previous studies [18] [19].

Table 9 lists the overall of $\mathrm{PM}_{2.5}$ mass balance closure, the measured $\mathrm{PM}_{2.5}$ masses agreed with the sums of the masses of the chemical components at all stations, except for ST1. In ST1, the measured PM 2.5 mass was almost $40 \%$ more than the mass sums of the chemical components. The house discrepancy could be due to uncertainty in the chemical composition measurements. The heavy PM loading on the filters (PM thickness) from ST1 could have big influence on the accuracy of the technique of X-ray fluorescence. Also the presence of bounded water on the filter could cause significant mass discrepancy. There was significant seasonal dependence of the mass balance discrepancy in ST1 (Figure 8(a)). In spring and winter, the measured PM $_{2.5}$ mass exceeded the mass sum of the chemical components with more than 50\%. As reported by [20] [21], there were much higher in-house PM concentrations in spring and winter than summer and autumn. The high PM concentrations could cause heavy filter loading, then cause large measurement uncertainty for element concentrations and mass fraction determination. For ambient stations, there was no significant seasonal dependence (Figure 8(b)).

\section{Conclusion}

This study determined chemical compositions of $\mathrm{PM}_{2.5}$ emitted from a commercial layer farm and in its vicinity in North Carolina. It was discovered that there were significant differences in elemental compositions between PM samples from in-house station (ST1) and ambient stations (ST2-ST5). Organic carbon accounted for above $50 \%$ of $\mathrm{PM}_{2.5}$ mass at all stations. Moreover, $\mathrm{NH}_{4}^{+}, \mathrm{SO}_{4}^{2-}$, and $\mathrm{NO}_{3}^{-}$accounted for about $40.0 \%$ of the total $\mathrm{PM}_{2.5}$ mass in ambient locations and for only $12 \%$ of the total $\mathrm{PM}_{2.5}$ mass in house. The measured $\mathrm{PM}_{2.5}$ masses agreed with the sums of the masses of the chemical compositions at all stations except for the in-house station (i.e., ST1). At ST1, the sum of chemical components mass was almost $40 \%$ less than the measured $\mathrm{PM}_{2.5}$ mass, which may be due to XRF measurement uncertainty for heavy loaded filters and bound water on the filters. 


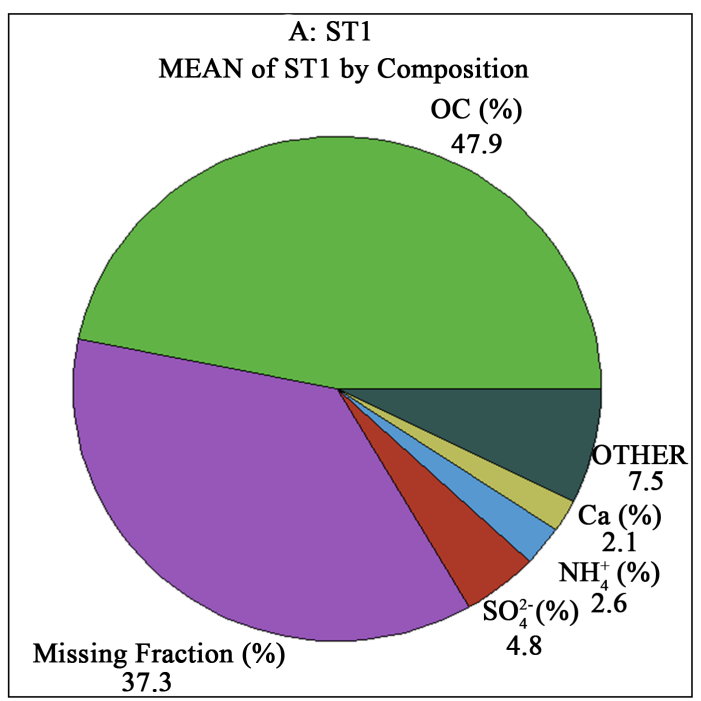

C: ST3

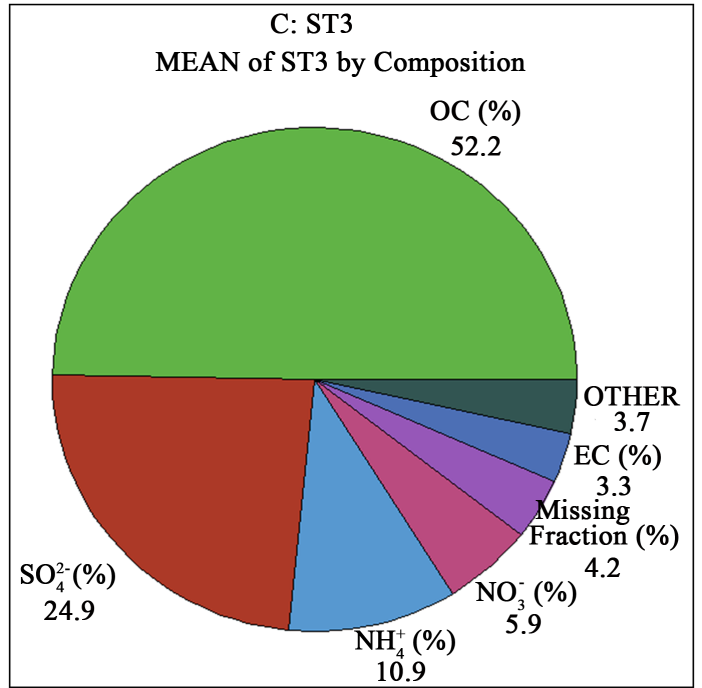

E: ST5

MEAN of ST5 by Composition

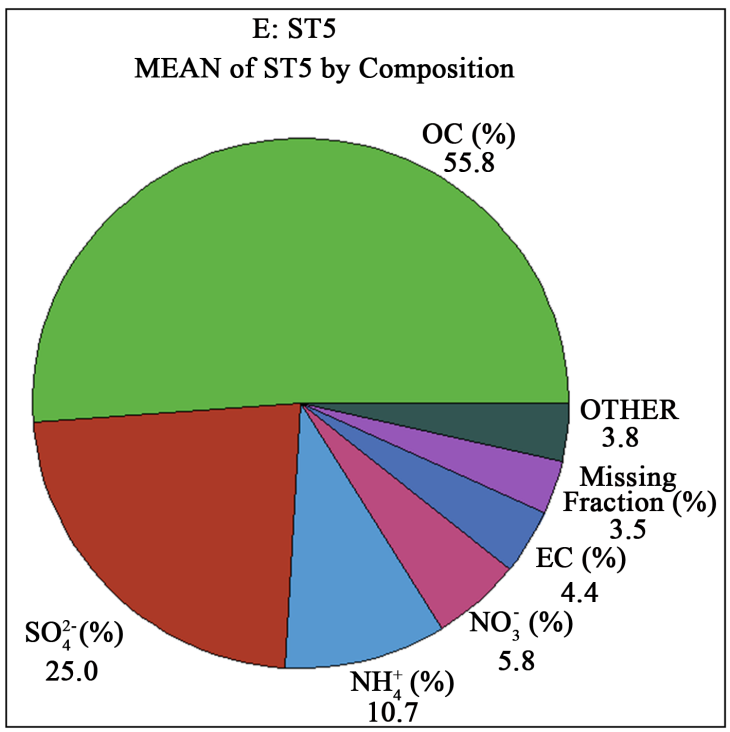

Figure 7. Average $\mathrm{PM}_{2.5}$ chemical compositions (\%). 


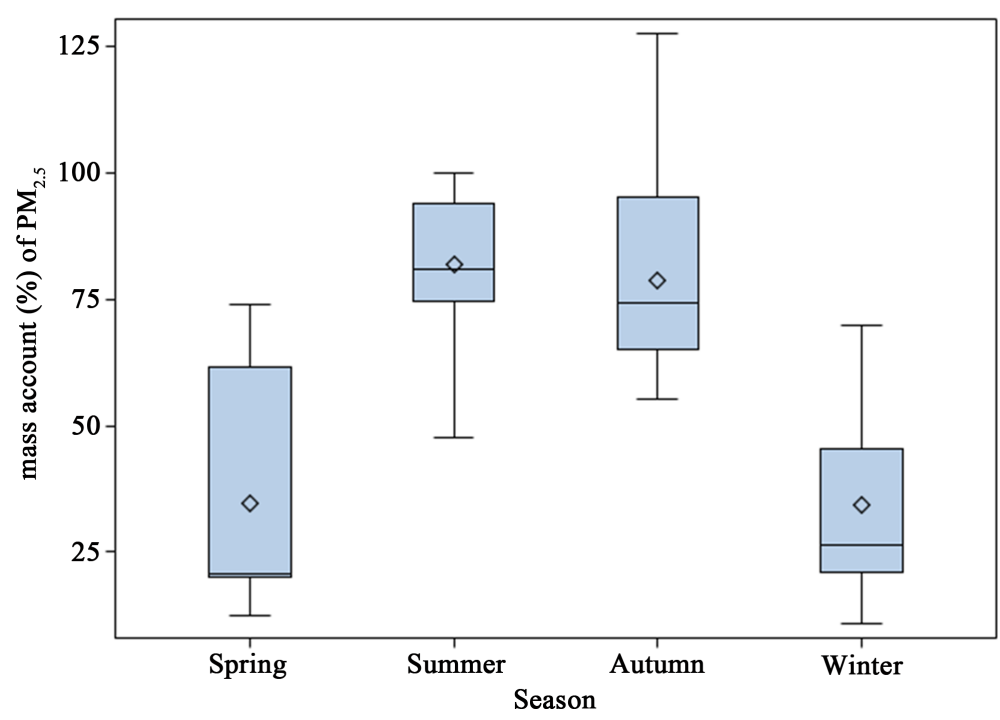

(a)

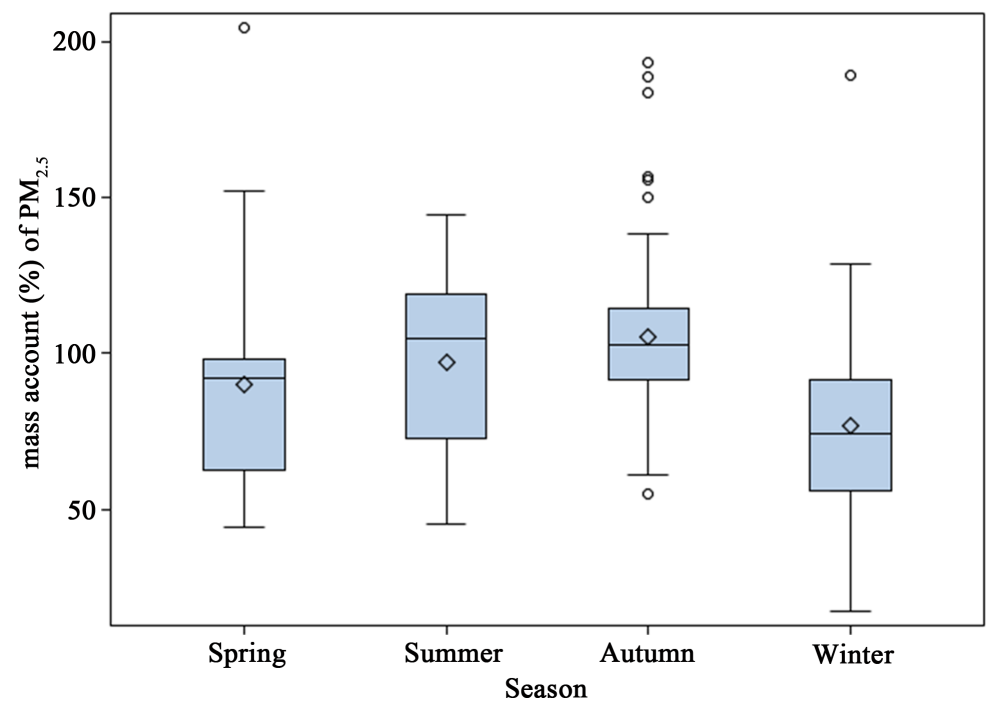

(b)

Figure 8. The seasonal $\mathrm{PM}_{2.5}$ mass balance. (a) = ST1 and (b) = ambient (average of ST2-ST5).

Table 9. $\mathrm{PM}_{2.5}$ mass balance closure.

\begin{tabular}{|c|c|c|c|c|c|c|c|c|}
\hline Station & $\mathrm{N}$ & Mean (\%) & Std Dev & Median & Min. & Maxi. & Lower 95\% CL for mean & Upper 95\% CL for mean \\
\hline ST1 & 63 & 62.7 & 28.8 & 69.4 & 10.8 & 127.6 & 55.5 & 70.0 \\
\hline ST2 & 61 & 92.0 & 26.0 & 93.1 & 46.9 & 189.3 & 85.4 & 98.7 \\
\hline ST3 & 63 & 95.8 & 29.8 & 94.7 & 44.1 & 193.1 & 88.3 & 103.3 \\
\hline ST4 & 64 & 97.2 & 28.4 & 97.8 & 44.8 & 183.4 & 90.1 & 104.3 \\
\hline ST5 & 66 & 96.5 & 29.2 & 99.4 & 17.2 & 204.4 & 89.3 & 103.6 \\
\hline
\end{tabular}

Knowledge gained from this study, with additional consideration of $\mathrm{NH}_{3}$ concentrations and emissions, will lead to better understanding of $\mathrm{PM}_{2.5}$ source and formation, fate and transport, and their atmospheric dynamics. 


\section{Acknowledgements}

This study was supported in part by the NSF-CAREER Award (No. CBET-0954673) and the National Research Initiative Competitive Grant No. 2008-35112-18757 from the USDA Cooperative State Research, Education, and Extension Service Air Quality Program and. The authors acknowledge the generous support and collaboration of the egg producer. Help from Zifei Liu with the field $\mathrm{PM}_{2.5}$ sample collection is thankfully acknowledged.

\section{References}

[1] Andersen, C.I., Von Essen, S.G., Smith, L.M., Spencer, J., Jolie, R. and Donham, K.J. (2004) Respiratory Symptoms and Airway Obstruction in Swine Veterinarians: A Persistent Problem. American Journal of Industrial Medicine, 46, 386-392. http://dx.doi.org/10.1002/ajim.20080

[2] Korrick, S.A., Neas, L.M., Dockery, D.W., Gold, D.R., Allen, G.A., Hill, L.B., Kimball, K.D., Rosner, B.A. and Speizer, F.E. (1998) Effects of Ozone and Other Pollutants on the Pulmonary Function of Adult Hikers. Environmental Health Perspectives, 106, 93-99. http://dx.doi.org/10.1289/ehp.9810693

[3] Kunzli, N. and Tager, I.B. (2000) Long-Term Health Effects of Particulate and Other Ambient Air Pollution: Research Can Progress Faster If We Want It to. Environmental Health Perspectives, 108, 915-918. http://dx.doi.org/10.1289/ehp.00108915

[4] Mitloehner, F.M. and Calvo, M.S. (2008) Worker Health and Safety in Concentrated Animal Feeding Operations. Journal of Agricultural Safety and Health, 14, 163-187. http://dx.doi.org/10.13031/2013.24349

[5] US EPA (2010) The Ambient Air Monitoring Program. http://epa.gov/airquality/qa/monprog.html

[6] US EPA (2010) Health and Environment. http://www.epa.gov/PM/health.html

[7] Hinds, W.C. (1998) Aerosol Technology: Properties, Behavior and Measurement of Airborne Particles. John Wiley \& Sons, Hoboken.

[8] US EPA (1997) Guidance for Network Design and Optimum Site Exposure for $\mathrm{PM}_{2.5}$ and $\mathrm{PM}_{10}$. Research Triangle Park, NC. http://www.epa.gov/ttn/amtic/files/ambient/pm25/network/r-99-022.pdf

[9] Kerminen, V.M., Hillamo, R., Teinila, K., Pakkanen, T., Allegrini, I. and Sparapani, R. (2001) Ion Balances of SizeResolved Tropospheric Aerosol Samples: Implications for the Acidity and Atmospheric Processing of Aerosols. Atmospheric Environment, 35, 5255-5265. http://dx.doi.org/10.1016/S1352-2310(01)00345-4

[10] Liang, J.Y. and Jacobson, M.Z. (1999) A Study of Sulfur Dioxide Oxidation Pathways over a Range of Liquid Water Contents, pH Values, and Temperatures. Journal of Geophysical Research-Atmospheres, 104, 13749-13769. http://dx.doi.org/10.1029/1999JD900097

[11] Yang, X., Wang, X., Zhang, Y., Lee, J., Su, J. and Gates, R.S. (2011) Characterization of Trace Elements and Ions in $\mathrm{PM}_{10}$ and $\mathrm{PM}_{2.5}$ Emitted from Animal Confinement Buildings. Atmospheric Environment, 45, 7096-7104. http://dx.doi.org/10.1016/j.atmosenv.2011.09.037

[12] Cambra-López, M., Toores, A.G., Aarnink, A.J.A. and Ognik, N.W.M. (2011) Source Analysis of Fine and Coarse Particulate Matter from Livestock Houses. Atmospheric Environment, 45, 694-707. http://dx.doi.org/10.1016/j.atmosenv.2010.10.018

[13] Cambra-López, M., Hermosilla, T., Lai, H.T., Aarnink, A.J.A. and Ognik, N.W.M. (2011) Particulate Matter Emitted from Poultry And Pig Houses: Source Identification and Quantification. Transactions of the ASABE, 54, 629-642. http://dx.doi.org/10.13031/2013.36466

[14] Wang-Li, L., Li, Q.-F., Wang, K., Bogan, B.W., Ni, J.-Q., Cortus, E.L. and Heber, A.J. (2013) The National Air Emissions Monitoring Study's Southeast Layer Site: Part I. Site Characteristics and Monitoring Methodology. Transaction of the ASABE, 56, 1157-1171.

[15] RTI International (2010) $\mathrm{PM}_{2.5}$ Chemical Speciation. Research Triangle Park, North Carolina. http://www.rti.org/page.cfm/PM_25_Chemical_Speciation

[16] USDA (2011) United States Department of Agriculture: Economics, Statistics, and Market Information System. http://usda.mannlib.cornell.edu/MannUsda/homepage.do

[17] Vucemilo, M., Matkovic, K., Vinkovic, B., Jaksic, S., Granic, K. and Mas, N. (2007) The Effect of Animal Age on Air Pollutant Concentration in a Broiler House. Czech Journal of Animal Science, 52, 170-174.

[18] Al Homidan, A., Robertson, J.F. and Petchey, A.M. (1998) Effect of Environmental Factors on Ammonia and Dust Production and Broiler Performance. British Poultry Science, 39, S9-S10.

[19] Tolocka, M.P., Solomon, P.A., Mitchell, W., Norris, G.A., Gemmill, D.B., Wiener, R.W., Vanderpool, R.W., Homolya, J.B. and Rice, J. (2001) East versus West in the US: Chemical Characteristics of PM2.5 during the Winter of 1999. Aerosol Science and Technology, 34, 88-96. http://dx.doi.org/10.1080/02786820118957 
[20] Li, Q.-F., Wang-Li, L., Walker, J.T., Shah, S.B., Bloomfield, P. and Jayanty, R.K.M. (2012) Particulate Matter in the Vicinity of an Egg Production Facility: Concentrations, Statistical Distributions and Upwind and Downwind Comparison. Transaction of the ASABE, 55, 1965-1973. http://dx.doi.org/10.13031/2013.42359

[21] Li, Q.-F., Wang-Li, L., Wang, K., Chai, L., Cortus, E.L., Kilic, I., Bogan, B.W., Ni, J.-Q. and Heber, A.J. (2013) National Air Emissions Monitoring Study's Southeast Layer Site: Part II. Particulate matter. Transaction of the ASABE, 56, 1173-1184. 